\title{
Adjunctive aripiprazole in risperidone-induced hyperprolactinaemia: double-blind, randomised, placebo-controlled trial
}

\section{G. Raghuthaman, R. Venkateswaran and R. Krishnadas}

\section{Background}

Hyperprolactinaemia is a troublesome side-effect of treatment with antipsychotics.

\section{Aims}

This double-blind, placebo-controlled study aimed at examining the effect of adjunctive treatment with $10 \mathrm{mg}$ aripiprazole on prolactin levels and sexual side-effects in patients with schizophrenia symptomatically maintained on risperidone.

\section{Method}

Thirty patients taking risperidone were enrolled into the trial (CTRI/2012/11/003114). Aripiprazole was administered at a fixed daily dose of $10 \mathrm{mg} /$ day for 8 weeks. Serum prolactin was measured at baseline and at 8 weeks. Hyperprolactinaemiarelated problems, psychopathology and side-effects were evaluated every 2 weeks.

\section{Results}

Prolactin levels decreased by $58 \%$ in the aripiprazole group compared with an increase by $22 \%$ in the placebo group.
Prolactin levels normalised in $46 \%$ of patients in the aripiprazole group (number needed to treat, NNT=2). Aripiprazole improved erectile dysfunction in five out of six patients. There were no significant differences in change in psychopathology or side-effects between groups

\section{Conclusions}

Adjunctive aripiprazole reduced prolactin levels in those treated with risperidone, with no effect on psychopathology and extrapyramidal symptoms. This is a potential treatment for hyperprolactinaemia observed during treatment with secondgeneration antipsychotics.

\section{Declaration of interest}

None.

\section{Copyright and usage}

(c) The Royal College of Psychiatrists 2015. This is an open access article distributed under the terms of the Creative Commons Non-Commercial, No Derivatives (CC BY-NC-ND) licence.
The lactotrophic cells of the anterior pituitary secrete high levels of prolactin following dopaminergic antagonism, even in the absence of any hormonal action. ${ }^{1}$ Hyperprolactinaemia is hence a worrisome side-effect of antipsychotic medications that inhibit $\mathrm{D}_{2}$ receptors in the tuberoinfundibular and hypophyseal pathways. Consequently, a proportion of people treated with antipsychotics develop galactorrhoea, amenorrhoea, gynaecomastia, erectile dysfunction, anorgasmia and osteoporosis in the long term. ${ }^{2-7}$ Hyperprolactinaemia and its consequences are therefore common reasons for non-adherence and treatment discontinuation. ${ }^{8}$ Although older first-generation antipsychotics (typical) were initially thought to be the culprits, second-generation antipsychotics (atypical) have also been implicated. Leucht et al in a metaanalysis comparing the tolerability of 15 antipsychotics found that most second-generation antipsychotics increased serum prolactin levels significantly. ${ }^{9}$ Given that most clinicians chose an antipsychotic based on efficacy, risperidone is one of the most preferred second-generation antipsychotics. ${ }^{10}$ However, hyperprolactinaemia is evidenced in three-quarters of patients receiving risperidone, both the oral and long-acting intramuscular preparations. $^{5,10,11}$ Around $45 \%$ of those with hyperprolactinaemia develop symptoms pertaining to hyperprolactinaemia. ${ }^{2}$ To manage these side-effects, clinicians either switch to a prolactinsparing antipsychotic medication or use dopamine agonists such as amantadine or bromocriptine. ${ }^{12-14}$ These strategies are not always successful and have been associated with a risk of relapse in psychotic symptoms. Among the newer antipsychotics, aripiprazole has been found to lower serum prolactin levels - albeit nonsignificantly. ${ }^{9}$ Aripiprazole is a high-affinity, low intrinsic activity, functionally selective partial $\mathrm{D}_{2}$ agonist. ${ }^{15}$ Natesan et al found that $\mathrm{D}_{2}$ receptor occupancy of aripiprazole at clinically effective doses are typically more than $90 \%$ (compared with $65 \%$ for most other antipsychotics). They found that in animal models haloperidol and risperidone administration (with $>80 \%$ occupancy) was associated with catalepsy and hyperprolactinaemia, whereas aripiprazole administration was not associated with these signs. They proposed that this dissociation between occupancy and functional antagonism was because of the partial agonism of aripiprazole. ${ }^{16}$ More recent research suggest that aripiprazole's functional effects depend on the cellular location and signalling proteins of the targeted $\mathrm{D}_{2}$ receptor. ${ }^{17}$ This functional selectivity seems to be associated with lack of hyperprolactinaemia and indeed normalisation/reduction of prolactin levels in certain situations. ${ }^{1,15,18}$

To date, three double-blind, placebo-controlled studies have explored the use of aripiprazole in treating antipsychotic-induced hyperprolactinaemia. ${ }^{19-22}$ The first study examined aripiprazole augmentation in haloperidol-induced hyperprolactinaemia; the second in risperidone-induced hyperprolactinaemia in males; and a third study exploring aripiprazole augmentation of risperidone/quetiapine reported reduction in prolactin levels as a secondary outcome measure. A recent meta-analysis found that these studies lacked details of random sequence generation, allocation concealment and masking. ${ }^{19}$ In addition, two of them did not report on prolactin-related symptom recovery. ${ }^{20,21}$

We therefore conducted an 8-week double-blind, randomised, placebo-controlled trial of $10 \mathrm{mg}$ of adjunctive aripiprazole in patients with schizophrenia maintained on a stable dose of risperidone (median dose $6 \mathrm{mg}$ ). Our primary outcome measures were serum prolactin levels and symptoms related to hyperprolactinaemia. Our secondary outcome measures were changes in psychopathology and emergent side-effects pertaining to this 
augmentation. We report our findings in accordance with the CONSORT guidelines. ${ }^{23}$

\section{Method}

The study was a single-centre double-blind, randomised, placebocontrolled trial conducted in the Department of Psychiatry, PSG Institute of Medical Sciences and Research (PSGIMSR), a medical college hospital in India. The study was approved by the institutional review board at PSGIMSR. All participants gave written informed consent. Details of the trial are registered on www.ctri.nic.in (Clinical Trials Registry - India: CTRI/2012/11/ 003114).

\section{Sample size calculation}

Our sample size calculation was based on Shim et al who explored the effect of aripiprazole on those treated with haloperidol. ${ }^{22}$ They found that at the end of 8 weeks, hyperprolactinaemia had normalised in $86 \%$ of those in the aripiprazole group compared with $3.6 \%$ of those in the placebo group. Using these proportions, we found that to observe a significant group difference with $90 \%$ power with an alpha of 0.01 (two-tailed), we needed a sample size of 11 in each group. Considering a drop-out rate of about $30 \%$, we required a sample size of 15 in each group. (For details of power calculation, see online supplement material.) The trial was stopped when recruitment was completed.

\section{Participants}

Between 1 January 2010 and 31 December 2010, 192 out of 564 patients with schizophrenia were being prescribed risperidone. From 1 January 2011, out of 50 participants screened for eligibility, we (G.R. and R.V.) enrolled 30 consecutive patients who met our inclusion criteria. Details are shown in the CONSORT flow diagram (Fig. 1). We included patients aged 15-45 years who fulfilled the DSM-IV criteria for schizophrenia using the Structured Clinical Interview for DSM-IV (SCID); ${ }^{24}$ patients were being prescribed a stable dose of risperidone at least for 12 weeks and were able to visit our centre once in 2 weeks to collect medications and for assessments. We excluded: (a) those who were receiving depot antipsychotics or any oral antipsychotics other than risperidone; (b) those with a history of neurological or any active medical illness; (c) pregnant or lactating mothers; and (d) those with a history of alcohol or other substance use disorder.

\section{Randomisation, masking and procedure}

Participants were screened and enrolled into the study by G.R. and R.V. Thirty participants were randomly assigned to either aripiprazole or control (placebo) group with a $1: 1$ allocation as per a computer-generated randomisation schedule using permuted blocks of random sizes, generated at the pharmacy. The block sizes were not disclosed to ensure concealment. Allocation concealment was ensured as the randomisation code was not released until all the recruitment and assessments were completed.

Aripiprazole (the study drug) was administered at a daily fixed dose of $10 \mathrm{mg}$ over a period of 8 weeks. To ensure masking, placebo tablets that were identical to the aripiprazole tablets were dispensed in 15 similar-looking sealed glass containers to the participants from the pharmacy. The investigators, participants and their carers were masked to the medications throughout the study and the code for allocation was kept within the pharmacy and broken only at the end of study.

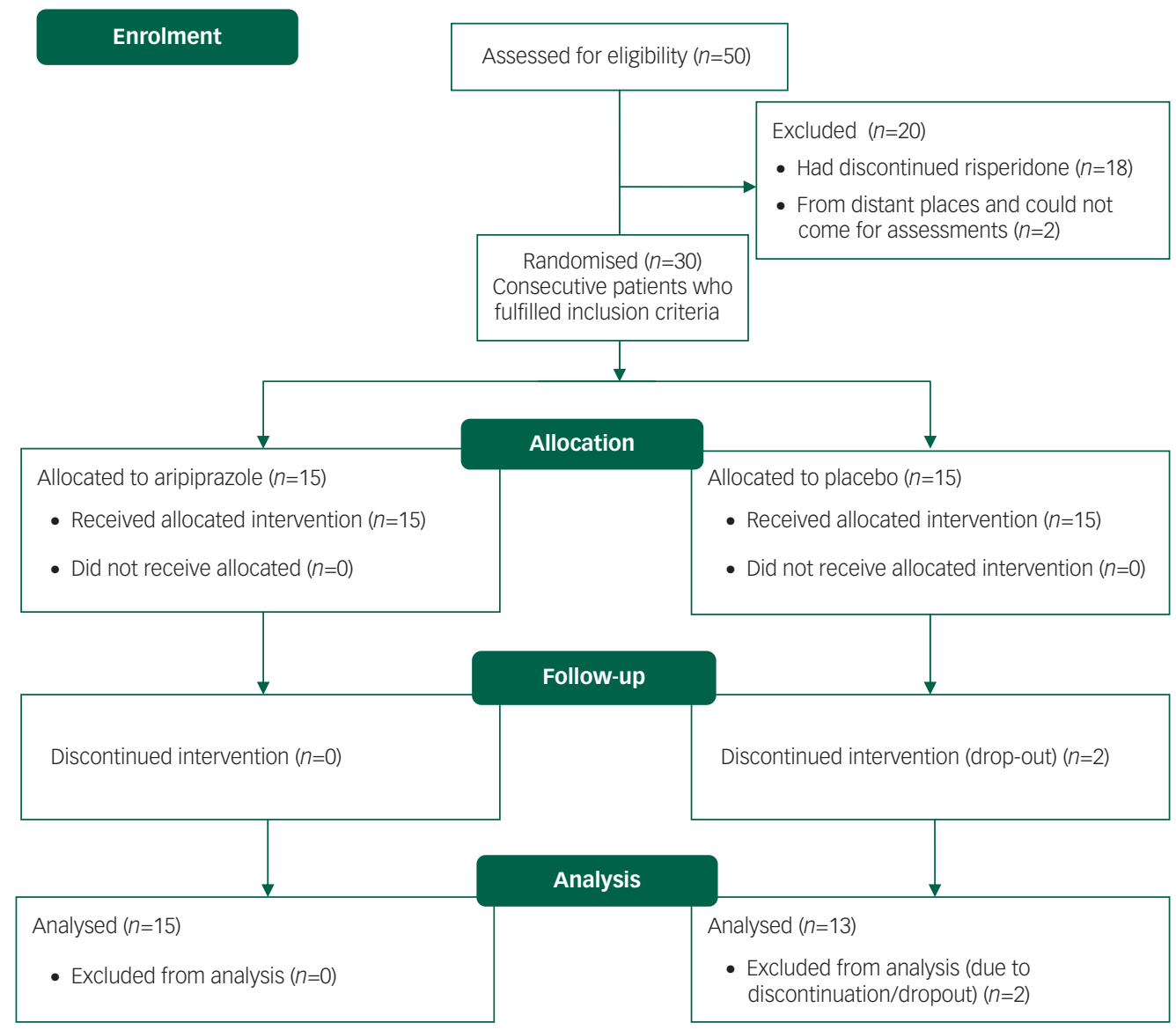

Fig. 1 CONSORT flow diagram. 


\section{Outcomes}

Rating scales

All rating scales were administered by an investigator (R.V.) who was masked to the treatment allocation at baseline, at 2-week intervals and at the end of the study. Severity of the psychosis was assessed using the Brief Psychiatric Rating Scale (BPRS). ${ }^{25}$ Neurological side-effects were monitored using the SimpsonAngus Extrapyramidal Symptom Rating Scale (SAES) for extrapyramidal side-effects and Barnes-Akathisia Rating Scale (BARS). ${ }^{26,27}$ Prolactin-related sexual and menstrual problems were evaluated using the Arizona Sexual Experience Scale ${ }^{28}$ and Prolactin-Related Adverse Event Questionnaire. ${ }^{29}$

\section{Prolactin measurement}

Prolactin levels were measured at baseline and at the end of 8 weeks. An early morning 12-hour fasting sample of blood was drawn from participants who were also asked to abstain from sexual intercourse on the previous night. Plasma prolactin level was measured by electrochemiluminescence immunoassay (ECLIA) based on quantitative sandwich immunoassay (Roche). The minimal detectable concentration of human prolactin by this assay is estimated to be $1.5 \mu \mathrm{IU} / \mathrm{mL}$. The monoclonal antibodies used are highly specific for prolactin and exhibits no detectable cross-reaction with human follicle-stimulating hormone, leutinising hormone, thyroid-stimulating hormone, chorionic gonadotropin and growth hormone. This immunoassay was calibrated against the World Health Organization's Third International Standard for Prolactin (preparation in ampoules coded 84/500) ${ }^{30}$ and no hook effect observed up to $10000 \mu \mathrm{IU} / \mathrm{mL}$. Hyperprolactinaemia was defined as the elevation of serum prolactin level $>324 \mu \mathrm{IU} / \mathrm{mL}$ in men or $>496 \mu \mathrm{IU} / \mathrm{mL}$ in women.

\section{Statistical analysis}

We tested for normality of continuous variables by examining their histograms and the Shapiro-Wilks test. Age, BPRS scores and prolactin levels were positively skewed. These variables were therefore log-transformed. All further parametric statistical analyses were done on these log-transformed scores. For the variables where transformation was not appropriate, we used non-parametric tests. For prolactin levels, BPRS and SAES, we performed a repeated measures analysis of variance (ANOVA) with time points (baseline $v$. follow-up) as within-group factor and medication group (aripiprazole $v$. placebo) as between-group factor. Effect sizes were calculated according to Cooper et al. ${ }^{31}$ When exploring follow-up/post hoc tests, all $P$-values were corrected for multiple testing using the Bonferroni method. Difference in baseline variables because of random chance was tested using unpaired $t$-tests and chi-squared tests. All reported $P$-values are two-tailed.

\section{Results}

Table 1 shows that none of the baseline variables were significantly different between groups.

\section{Prolactin levels}

On repeated measures ANOVA, there was a significant effect of time $(F(1,26)=22.058 ; P<0.001)$, group $(F(1,26)=13.24 ; P=0.001)$ and a significant group $\times$ time interaction $(F(1,26)=33.54$; $P<0.001$; Fig. 2).

\section{Difference between medications - aripiprazole $v$. placebo}

Following up the group $\times$ time interaction, there was no difference in prolactin levels between the two groups at baseline (mean difference 704.46; $P=0.1$ Bonferroni corrected; $d=0.66 ; 95 \% \mathrm{CI}$ -0.09 to 1.42 ). However, the difference between the two groups were significant at follow-up (mean difference 1616.19; $P<0.001$; Bonferroni corrected; $d=2.05$; 95\% CI 1.13 to 2.96).

\section{Difference between baseline and 8-week follow-up - change in prolactin levels}

Over the 8-week period, there was a significant reduction in prolactin levels in the aripiprazole group (mean difference 853.73; $P<0.001$; Bonferroni corrected; $d=1.04 ; 95 \%$ CI 0.67 to 1.63 ). Prolactin levels fell by $58 \%$ in the aripiprazole group. There was no reduction in prolactin levels in the placebo group (mean difference $=-43.85 ; \quad P=0.46 ; \quad$ Bonferroni corrected; $d=-0.14$; $95 \%$ CI -0.59 to 0.13 ). Prolactin levels increased by $22 \%$ in the placebo group.

\section{Analysis with baseline prolactin level and gender as covariate in the} model

Although there was no significant difference in baseline prolactin level between groups, there was a significant association between baseline prolactin levels and change in prolactin levels $(r=-0.4$; $P=0.05)$. We therefore repeated the analysis with baseline prolactin levels as covariate in the above model. The group $\times$ time interaction remained significant $(F(1,25)=37.29 ; P<0.001$; see Fig. DS1). In addition, prolactin levels were greater in women compared with men $(t=7.56 ; P<0.001)$. Although there was no difference in the number of men between the two groups, we repeated the analysis with gender as a covariate in the model. Once again, the group $\times$ time interaction remained significant $(F(1,25)=31.24 ; P<0.001)$.

\section{Hyperprolactinaemia}

The percentage of people who fulfilled the criteria for hyperprolactinaemia did not differ between groups at baseline (Table 1). Whereas all but one patient in the aripiprazole group showed a reduction in prolactin levels, only 4 out of 13 patients in the placebo group showed a reduction (Fisher's exact test, $P=0.004$ ).

\begin{tabular}{|c|c|c|c|c|}
\hline & Aripiprazole & Placebo & Tests & $P$ (two-tailed) \\
\hline Age, years: mean (s.d.) & $32.4(7.48)$ & $32.07(9.23)$ & $t=0.108^{a}$ & 0.91 \\
\hline BPRS score, mean (s.d.) & $9.6(5.81)$ & $9.00(5.33)$ & $t=0.11^{\mathrm{a}}$ & 0.91 \\
\hline SAES score, mean (s.d.) & $3.27(2.052)$ & $3.73(1.438)$ & $t=-0.72$ & 0.47 \\
\hline Male, $n(\%)$ & 9 & 6 & $\chi^{2}=0.27$ & 0.47 \\
\hline Risperidone dose, mg: median (IQR) & $6(0)$ & $6(0)$ & $U=93^{b}$ & 0.44 \\
\hline Hyperprolactinaemia, $n$ (\%) & $13(86)$ & $15(100)$ & $\chi^{2}=0.53$ & 0.46 \\
\hline Prolactin levels, $\mu \mathrm{IU} / \mathrm{mL}$ : mean (s.d.) & $1408(1336)$ & $2112(1648.3)$ & $t=-1.72^{\mathrm{a}}$ & 0.1 \\
\hline \multicolumn{5}{|c|}{$\begin{array}{l}\text { BPRS, Brief Psychiatric Rating Scale; SAES, Simpson-Angus Extrapyramidal Symptom Rating Scale. } \\
\text { a. Unpaired } t \text {-tests based on log-transformed data. } \\
\text { b. Mann-Whitney U-test. }\end{array}$} \\
\hline
\end{tabular}




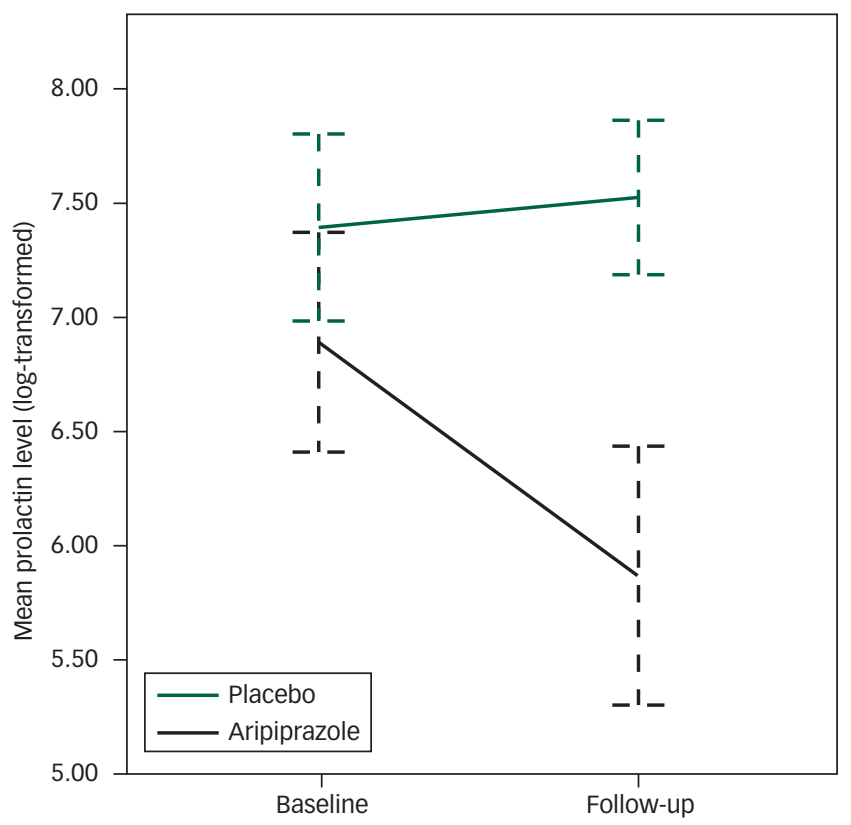

Fig. 2 Change in mean prolactin level (log-transformed) from baseline to 8-week follow-up in the aripiprazole and the placebo group Error bars represent $95 \% \mathrm{Cl}$.

At follow-up, the prolactin level had normalised in $46.7 \%$ of those treated with aripiprazole compared with none in the placebo group (Fisher's exact test, $P=0.007$ ). The number needed to treat (NNT) was 2.1.

\section{Prolactin-related symptoms}

Between 63 and $80 \%$ of patients had sexual dysfunctions at baseline (Table 2).

\section{Men}

Six (66.7\%) men in the aripiprazole group and four (66\%) in the placebo group had erectile dysfunction. Aripiprazole improved erectile dysfunction in five out of six patients. In contrast, one additional patient developed erectile dysfunction in the placebo group at follow-up. The difference in the proportion of men with erectile dysfunction at follow-up between the two medication groups was statistically significant (Fisher's exact, $P=0.01$ ).

\section{Women}

Menstrual problems did not change in the aripiprazole group at 8 weeks; $83.3 \%$ of women continued to have menstrual irregularities. Among the whole sample, at baseline, $67.7 \%$ of women had fluid at nipples and milk staining of underclothes and this fell to $14.2 \%$ at the end of 8 weeks with no difference between the two groups.

\section{Adverse effects}

Four patients in the aripiprazole group developed adverse events Two patients showed a worsening of psychotic symptoms. These symptoms remitted with the addition of lorazepam. Two patients showed symptoms suggestive of gastritis (which required no treatment). Two patients in the placebo group developed adverse effects. One of them developed insomnia and the other developed infectious hepatitis (which was unlikely to be associated with the trial).

\section{Psychopathology and side-effect ratings}

There were no significant changes in the severity of psychopathology as measured by the BPRS (group $\times$ time interaction; $F(1,26)=$ $0.75 ; P=0.75$ ) and adverse effects as rated by the SAES (group $\times$ time interaction; $F(1,26)=0.55 ; P=0.46)$. All participants scored 0 at baseline and follow-up on the BARS.

\section{Discussion}

We have shown using a randomised, double-blind, placebocontrolled trial that adjunctive aripiprazole is a safe, practical and effective option in risperidone-induced hyperprolactinaemia. In summary, prolactin levels decreased by $58 \%$ in the aripiprazole group compared with an increase by $22 \%$ in the placebo group. Prolactin levels had normalised in $46 \%$ of the participants in the aripiprazole group compared with none in the placebo group. The NNT was 2.1, i.e. about two patients with hyperprolactinaemia would have to be treated with aripiprazole for one additional patient's hyperprolactinaemia to normalise. More importantly, we showed that this reduction in prolactin level was associated with an improvement in erectile dysfunction in five out of six patients. In contrast, one additional patient developed erectile dysfunction in the placebo group at follow-up.

Why does aripiprazole have a prolactin neutralising effect? Drugs such as clozapine and quetiapine are thought to have a favourable effect on prolactin levels because of their fast dissociation from $\mathrm{D}_{2}$ receptors. ${ }^{32}$ Interestingly, aripiprazole in spite of having high affinity and slow dissociation from $\mathrm{D}_{2}$ receptors, shows prolactin-sparing properties. Although aripiprazole was classically described as a partial agonist, it is now known that its intrinsic action depends on the signalling environment of the $\mathrm{D}_{2}$ receptors, indicative of functional selectivity. ${ }^{1,18}$ In other words, the mechanism of action of aripiprazole (whether it acts as an agonist/partial agonist/antagonist) depends on the location and the second messenger system associated with the $\mathrm{D}_{2}$ receptor to which it binds. ${ }^{17}$ Therefore, aripiprazole seems to be an antagonist at the mesocorticolimbic $\mathrm{D}_{2}$ receptors, whereas the lack of cataleptogenic effects suggests partial agonist activity at the striatal $\mathrm{D}_{2}$ receptors. ${ }^{15,33}$ Similarly, the ability of aripiprazole to suppress serum prolactin in humans is likely to be mediated by partial agonism at the anterior pituitary $\mathrm{D}_{2}$ receptors. ${ }^{33}$ In addition to these mechanisms, aripiprazole is an agonist at 5HT1 and antagonist at 5HT2A receptors, which may contribute to its prolactin neutral effect. ${ }^{1}$

Our findings are in keeping with previous reports of aripiprazole-induced reduction in prolactin levels. ${ }^{9}$ Shim et al explored the use of high dose $(15 \mathrm{mg}$ for the first 4 weeks and $30 \mathrm{mg}$ following that) aripiprazole in haloperidol-induced hyperprolactinaemia in an 8-week randomised, double-blind, placebo-controlled trial. ${ }^{22}$ They found that prolactin levels normalised in $88.5 \%$ of individuals with an $84.2 \%$ reduction in prolactin level. This was well above the normalisation rate/ reduction seen in our study. Although this could be attributed to the lower dose of aripiprazole used in our study, a second study by Chen et al that used just $5 \mathrm{mg}$ of aripiprazole in males prescribed risperidone also found greater prolactin normalisation (67.6\%) compared with our study. ${ }^{20}$ Interestingly, a third study by Kane et al (10 mg aripiprazole for augmentation) that reported changes in prolactin levels as a secondary outcome measure in patients treated with risperidone or quetiapine found lower prolactin normalisation rate $(19.5 \%)$ compared with our study. ${ }^{21}$ Taken together, these findings suggest that the dose differences in aripiprazole may not explain the difference in normalisation rates. 


\begin{tabular}{|c|c|}
\hline & $\begin{array}{l}\text { People reporting } \\
\text { symptoms, \% }\end{array}$ \\
\hline \multicolumn{2}{|l|}{ Sexual experiences } \\
\hline Perceiving their sex as weak & 76.7 \\
\hline Difficulty in getting sexually aroused & 63.3 \\
\hline $\begin{array}{l}\text { Difficulty in getting and maintaining } \\
\text { erection (men) }\end{array}$ & 66.7 \\
\hline $\begin{array}{l}\text { Difficulty in making the vagina moist } \\
\text { (women) }\end{array}$ & 80 \\
\hline \multicolumn{2}{|l|}{ Galactorrhoea } \\
\hline Men & 0 \\
\hline \multicolumn{2}{|l|}{ Women } \\
\hline $\begin{array}{l}\text { Perception of increased breast size and } \\
\text { tenderness }\end{array}$ & 6.7 \\
\hline Perception of fluid at nipples & 40 \\
\hline Perception of staining under clothes & 27.7 \\
\hline \multicolumn{2}{|l|}{ Menstrual changes } \\
\hline $\begin{array}{l}\text { Having longer time between their periods } \\
\text { and fewer days of menstrual flow }\end{array}$ & 79.9 \\
\hline
\end{tabular}

However, there is some evidence to suggest that the prolactin normalising effect of aripiprazole may be dose-dependent. ${ }^{34}$

Our results suggest that reduction in prolactin was independent of baseline levels and gender, making aripiprazole a feasible option in the treatment of hyperprolactinaemia in both men and women. Although previous trials examined prolactin levels, only Shim et al reported the effect of aripiprazole on sexual sideeffects. $^{22}$ This was in the context of treatment with haloperidol, and they did not comment on sexual dysfunction in males. In our study, most of the men had an improvement in erectile dysfunction symptoms. This is consistent with previous studies that found aripiprazole to be associated with lower sexual symptoms compared with other antipsychotics. In women, unlike Shim et $a l$, we found that menstrual symptoms persisted in most of our patients. ${ }^{22}$ This perhaps is because although there was a significant reduction, the prolactin levels remained well above the normal range (mean $1097 \mathrm{IU}$ ) in women. Once again, this could be attributed to the lower dose of aripiprazole in our study compared with Shim et $a l^{22}$ It is also possible that continued treatment may have led to greater normalisation of hyperprolactinaemia and reversal of these symptoms over time. Although menstrual symptoms showed no change, galactorrhoea improved in the aripiprazole group. As seen in previous studies, none of the men complained of galactorrhoea. Galactorrhoea is a rare symptom of hyperprolactinaemia in males. ${ }^{6,35}$ Our findings are similar to that of Hanssens et al who found that more men reported improvement in sexual side-effects on treatment with aripiprazole compared with women. ${ }^{36}$ This may also explain why Chen et al reported greater normalisation rates at a smaller dose of aripiprazole, as theirs was an exclusively male sample. ${ }^{20}$ It is possible that different doses are required to treat hyperprolactinaemia in men and women. Longer follow-up periods (than two menstrual cycles in our case) may be required to ascertain improvement in menstrual symptoms.

In general, aripiprazole was well tolerated. Akathisia is a common side-effect with aripiprazole. However, we did not find any emergent akathisia in our sample. Extrapyramidal side-effects did not differ between the groups at either baseline or follow-up. Psychotic symptoms worsened in two patients in the aripiprazole group. This is in keeping with previous reports of worsening psychotic symptoms in people treated with adjunctive or those being switched to aripiprazole. ${ }^{37}$ Previous studies have implicated high-dose antipsychotic therapy (chlorpromazine equivalent dose $>700 \mathrm{mg} /$ day) and discontinuation of previous antipsychotics as risk factors for emergent/worsening psychosis with aripiprazole. ${ }^{38}$ In our study, the median dose of risperidone was $6 \mathrm{mg} /$ day (chlorpromazine equivalent of around $400 \mathrm{mg} /$ day), far less than that implicated by Takeuchi et $a l^{38}$ As this was an adjunctivetreatment study, all our patients continued to receive risperidone throughout the study. Moreover, these symptoms were controlled by administering benzodiazepines. Although agonism pertaining to distinct conformations of upregulated $\mathrm{D}_{2}$ receptors has been implicated, the exact mechanism underlying emergent/worsening psychotic symptoms during aripiprazole augmentation remains unclear. $^{39}$

Our study had several limitations. Although we ensured that our sample size was based on power calculation, our trial was underpowered to conduct subgroup analysis. Even though we showed that our analysis was robust to differences in baseline prolactin levels and baseline gender differences in prolactin levels, further analysis to explore the differential effect of aripiprazole on men and women require large sample sizes. We examined a fixed dose of aripiprazole. Although meta-analysis of trials shows that the dose of aripiprazole does not seem to have an effect on the hypoprolactinaemic effect, a randomised, placebo-controlled trial with multiple doses will directly address this question. ${ }^{19}$ We explored the effect of aripiprazole on risperidone-induced hyperprolactinaemia. Although risperidone is one of the most commonly used medications, others such as sulpride and amisulpride are also associated with hyperprolactinaemia. ${ }^{10,40}$ There are some data to suggest that aripiprazole may be more effective in risperidone-induced hyperprolactinaemia compared with amisulpride-induced hyperprolactinaemia. ${ }^{41}$ Although the reasons for this specificity remain speculative, double-blind, placebo-controlled trials are essential in confirming whether aripiprazole is effective in treating amisulpride-induced hyperprolactinaemia. In addition, measurement of plasma levels of both aripiprazole and risperidone may have given us a better understanding of the mechanism underlying this effect. We also did not rule out prolactin-secreting tumours in our sample. Extremely high prolactin levels merit further investigation and treatment. Detailed guidelines of how to deal with this is provided elsewhere. ${ }^{42}$ Although we showed significant reductions in sexual side-effects in men, longer follow-up studies are needed to see whether these effects are sustained and whether menstrual symptoms improve in females.

In conclusion, we have shown that adjunctive aripiprazole treatment is a safe, practical and effective treatment option for patients with risperidone-induced high prolactin levels. It is relatively well tolerated. Long-term studies are warranted to ensure long-term safety of this treatment.

G. Raghuthaman, MBBS, DPM, MD, Department of Psychiatry, PSG Institute of Medical Sciences and Research, Coimbatore, India; R. Venkateswaran, MBBS, MD, Department of Child Psychiatry, CMC, Vellore, India; R. Krishnadas, MBBS, MD, MRCPsych, PhD, ESTEEM, NHS Greater Glasgow and Clyde, Glasgow, UK

Correspondence: Rajeev Krishnadas, Department of Psychiatry, ESTEEM, Glasgow G21 4SF, UK. Email: rajeev.krishnadas@glasgow.ac.uk

First received 15 Jun 2015, final revision 16 Sep 2015, accepted 17 Nov 2015

\section{Funding}

The authors acknowledge financial support from Indian Medical Council of Research (ICMR) India. The funder of the study had no role in study design, data collection, data analysis, data interpretation or writing of the report. 


\section{References}

1 Kucka M, Tomić M, Bjelobaba I, Stojilkovic SS, Budimirovic DB. Paliperidone and aripiprazole differentially affect the strength of calcium-secretion coupling in female pituitary lactotrophs. Sci Rep 2015; 5: 8902.

2 Melkersson K. Differences in prolactin elevation and related symptoms of atypica antipsychotics in schizophrenic patients. J Clin Psychiatry 2005; 66: 761-7.

3 Melkersson Kl. Prolactin elevation of the antipsychotic risperidone is predominantly related to its 9-hydroxy metabolite. Hum Psychopharmacol 2006; 21 529-32.

4 O'Keane V, Meaney AM. Antipsychotic drugs: a new risk factor for osteoporosis in young women with schizophrenia? J Clin Psychopharmacol 2005; 25: 26-31.

5 Volavka J, Czobor P, Cooper TB, Sheitman B, Lindenmayer JP, Citrome L, et al. Prolactin levels in schizophrenia and schizoaffective disorder patients treated with clozapine, olanzapine, risperidone, or haloperidol. J Clin Psychiatry 2004; 65: $57-61$.

6 Beumont PJ, Corker CS, Friesen HG, Kolakowska T, Mandelbrote M, Marshall J, et al. The effects of phenothiazines on endocrine function: II. Effects in men and post-menopausal women. Br J Psychiatry 1974; 124: 420-30.

7 Beumont PJ, Gelder MG, Friesen GH, Harris GW, MacKinnon PC, Mandelbrote BM, et al. The effects of phenothiazines on endocrine function: I. Patients with inappropriate lactation and amenorrhoea. Br J Psychiatry 1974; 124: 413-9.

8 Rosenberg KP, Bleiberg KL, Koscis J, Gross C. A survey of sexual side-effects among severely mentally ill patients taking psychotropic medications: impact on compliance. J Sex Marital Ther 2003; 29: 289-96.

9 Leucht S, Cipriani A, Spineli L, Mavridis D, Örey D, Richter F, et al. Comparative efficacy and tolerability of 15 antipsychotic drugs in schizophrenia: a multipletreatments meta-analysis. Lancet 2013; 382: 951-62.

10 Jauhar S, Guloksuz S, Andlauer O, Lydall G, Marques JG, Mendonca L, et al. Choice of antipsychotic treatment by European psychiatry trainees: are decisions based on evidence? BMC Psychiatry 2012; 12: 27

11 Peuskens J, Pani L, Detraux J, De Hert M. The effects of novel and newly approved antipsychotics on serum prolactin levels: a comprehensive review. CNS Drugs 2014; 28: 421-53.

12 Cavallaro R, Cocchi F, Angelone SM, Lattuada E, Smeraldi E. Cabergoline treatment of risperidone-induced hyperprolactinemia: a pilot study. J Clin Psychiatry 2004; 65: 187-90.

13 Stroup TS, McEvoy JP, Ring KD, Hamer RH, LaVange LM, Swartz MS, et al. A randomized trial examining the effectiveness of switching from olanzapine, quetiapine, or risperidone to aripiprazole to reduce metabolic risk: comparison of antipsychotics for metabolic problems (CAMP). Am J Psychiatry 2011; 168 947-56.

14 Lee MS, Song HC, An H, Yang J, Ko YH, Jung IK, et al. Effect of bromocriptine on antipsychotic drug-induced hyperprolactinemia: eight-week randomized, singleblind, placebo-controlled, multicenter study. Psychiatry Clin Neurosci 2010; 64 19-27.

15 Urban JD, Vargas GA, von Zastrow M, Mailman RB. Aripiprazole has functionally selective actions at dopamine $D_{2}$ receptor-mediated signaling pathways. Neuropsychopharmacology 2007; 32: 67-77.

16 Natesan S, Reckless GE, Nobrega JN, Fletcher PJ, Kapur S. Dissociation between in vivo occupancy and functional antagonism of dopamine $D_{2}$ receptors: comparing aripiprazole to other antipsychotics in animal models. Neuropsychopharmacology 2006; 31: 1854-63.

17 Mailman R, Murthy V. Third generation antipsychotic drugs: partial agonism or receptor functional selectivity? Curr Pharm Des 2010; 16: 488-501.

18 Shapiro DA, Renock S, Arrington E, Chiodo LA, Liu L-X, Sibley DR, et al. Aripiprazole, a novel atypical antipsychotic drug with a unique and robust pharmacology. Neuropsychopharmacology 2003; 28: 1400-11.

19 Li X, Tang Y, Wang C. Adjunctive aripiprazole versus placebo for antipsychoticinduced hyperprolactinemia: meta-analysis of randomized controlled trials. PLOS One 2013; 8: e70179.

20 Chen $H Z$, Niu FR, Qian MC, Yu BR, shen XH, Yang SG, et al. The effect of aripiprazole plus risperidone on the level of hyperprolactinemia of male patients with schizophrenia. Zhong Hua Jing Shen Ke Za Zhi 2009; 42: 224-7.
21 Kane JM, correll $C U$, Goff $D C$, Kirkpatrick $B$, Marder $S R$, Vester-Blokland $E$, et al. A multicenter, randomized, double-blind, placebo-controlled, 16-week study of adjunctive aripiprazole for schizophrenia or schizoaffective disorder inadequately treated with quetiapine or risperidone monotherapy. J Clin Psychiatry 2009; 70: $1348-57$.

22 Shim J-C, Shin JG, Kelly DL, Jung D-U, Seo Y-S, Liu K-H, et al. Adjunctive treatment with a dopamine partial agonist, aripiprazole, for antipsychotic-induced hyperprolactinemia: a placebo-controlled trial. Am J Psychiatry 2007; 164: 1404-10.

23 Moher D, Hopewell S, Schulz KF, Montori V, Gotzsche PC, Devereaux PJ, et al. CONSORT 2010 explanation and elaboration: updated guidelines for reporting parallel group randomised trials. BMJ 2010; 340: C869.

24 First MB, Spitzer RL, Gibbon M, Williams JBW. Structured Clinical Interview for Axis I DSM-IV Disorders. Biometrics Research, 1994.

25 Overall JE, Gorham DR. The brief psychiatric rating scale. Psychol Rep 1962: 10 799-812.

26 Simpson GM, Angus JW. A rating scale for extrapyramidal side-effects. Acta Psychiatr Scand Supp/ 1970; 212: 11-9.

27 Barnes TR. A rating scale for drug-induced akathisia. Br J Psychiatry 1989; 154 672-6.

28 McGahuey CA, Gelenberg AJ, Laukes CA, Moreno FA, Delgado PL, McKnight KM, et al. The Arizona Sexual Experience Scale (ASEX): reliability and validity. I Sex Marital Ther 2000; 26: 25-40.

29 Kelly DL, Conley RR. A randomized double-blind 12-week study of quetiapine, risperidone or fluphenazine on sexual functioning in people with schizophrenia. Psychoneuroendocrinology 2006; 31: 340-6.

30 Schulster D, Gaines Das RE, Jeffcoate SL. International Standards for human prolactin: calibration by international collaborative study. J Endocrinol 1989; 121 $157-66$

31 Cooper HM, Hedges LV, Valentine JC. The Handbook of Research Synthesis and Meta-analysis. Russell Sage Foundation, 2009.

32 Carboni L, Negri $M$, Michielin F, Bertani S, Fratte SD, Oliosi B, et al. Slow dissociation of partial agonists from the $D_{2}$ receptor is linked to reduced prolactin release. Int J Neuropsychopharmacol 2012; 15: 645-56.

33 Davies MA, Sheffler DJ, Roth BL. Aripiprazole: a novel atypical antipsychotic drug with a uniquely robust pharmacology. CNS Drug Rev. 2004; 10: 317-36.

34 Yasui-Furukori N, Furukori H, Sugawara N, Fujii A, Kaneko S. Dose-dependent effects of adjunctive treatment with aripiprazole on hyperprolactinemia induced by risperidone in female patients with schizophrenia. J Clin Psychopharmacol 2010; 30: 596-9.

35 Schlechte JA. Clinical practice. Prolactinoma. N Eng/ J Med 2003; 349: 2035-41.

36 Hanssens L, L'Italien G, Loze J-Y, Marcus RN, Pans M, Kerselaers W. The effect of antipsychotic medication on sexual function and serum prolactin levels in community-treated schizophrenic patients: results from the Schizophrenia Trial of Aripiprazole (STAR) study (NCT00237913). BMC Psychiatry 2008; 8: 95.

37 Takeuchi H, Remington G. A systematic review of reported cases involving psychotic symptoms worsened by aripiprazole in schizophrenia or schizoaffective disorder. Psychopharmacology (Berl) 2013; 228: 175-85.

38 Takeuchi $\mathrm{H}$, Uchida $\mathrm{H}$, Suzuki $\mathrm{T}$, Watanabe $\mathrm{K}$, Kashima $\mathrm{H}$. Predictors of clinical worsening after a switch to aripiprazole in patients with schizophrenia: a 1-year naturalistic follow-up study. J Clin Psychopharmacol 2009; 29: 394-5.

39 Gugger JJ, Tam CL, Ashby CR. Is there a link between aripiprazole and treatmentemergent psychosis? Curr Psychiatry 2011; 10: 53-8.

40 Manschreck TC, Boshes RA. The CATIE schizophrenia trial: results, impact, controversy. Harv Rev Psychiatry 2007; 15: 245-58.

41 Chen CK, Huang YS, Ree SC, Hsiao CC. Differential add-on effects of aripiprazole in resolving hyperprolactinemia induced by risperidone in comparison to benzamide antipsychotics. Prog Neuropsychopharmacol Biol Psychiatry 2010; 34: 1495-9.

42 Levy A. Interpreting raised serum prolactin results. BMJ 2014; 348: g3207.

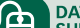

